\title{
Influence of Adopting a Text-Free User Interface on the Usability of a Web-based Government System with Illiterate and Semi-Literate People
}

\author{
Ms. Ghadam Alduhailan \\ Department of Information Systems \\ College of Computer Sciences and Information Technology \\ King Faisal University, Hofuf P.O. Box 380 Al-Ahsa \\ 31982 Kingdom of Saudi Arabia
}

\author{
Dr. Majed Alshamari \\ Department of Information Systems \\ College of Computer Sciences and Information Technology \\ King Faisal University, Hofuf P.O. Box 380 Al-Ahsa \\ 31982 Kingdom of Saudi Arabia
}

\begin{abstract}
Illiterate and semi-literate people usually face different types of difficulties when they use the Internet, such as reading and recognising text. This research aims to develop and examine the influence of adopting a text-free user interface on the usability of a web-based government system with illiterate and semi-literate people. A number of steps have been followed in order to achieve this research goal. An extensive literature review has been carried out to explore the adoption of different concepts or representations of content to help illiterate/semiliterate people in Information and Communication Technology (ICT) projects. Then a consolidated framework is proposed and adopted in order to develop a text-free user interface. This can help in building a text-free user interface for a certain service here in Saudi Arabia. Cultural factors, education level, text-free icons, and usability guidelines have been considered in the abovementioned framework. A prototype of a web-based government system after taking into account the above framework has been designed and developed. Usability testing and heuristic evaluation have been used as usability assessment methods in order to evaluate the system usability and its impact on the usability for illiterate people in Saudi Arabia. The results are encouraging as the achieved results of usability measures imply that adopting the consolidated framework has influenced the usability in this research.
\end{abstract}

Keywords-text-free interface; web-based system; usability; eservices; government; consolidated framework

\section{INTRODUCTION}

Information and communication technology (ICT) has improved societies in different ways. Actually, people consider technology to be a tool that can help them to perform their daily functions efficiently and effectively. E-governments use the World Wide Web (WWW) as a tool to deliver their services to all citizens.

Most e-government websites provide e-services that rely on textual interface conjunction with little use of graphics and icons that describe the content of websites. Therefore, a group of users can easily use and interact with governments' websites and take advantage of available services. However, there are groups of users in society who are not able to access and benefit from e-government services. Users who are unable to read and write struggle with the accessibility of available services due to the heavy use of text on most governmental websites. Different ways have been proposed in order to assess the usage of these users' categories, such as accessibility tools, usability evaluation methods, and other technologies.

Success in implementing and developing e-government is the goal of many government organisations. This process requires a good understanding of the needs of users and system requirements. Thus, such requirements are expected to be considered including illiterate or semi-literate, and even people who suffer from computer illiteracy.

The rest of the paper is organized as follows. Background and related work is discussed in section II. Section III presents the research questions and the used methodology in this research. It includes how the website was selected and the justifications. Participants recruitment and tasks' selections are also explained in this section. Data analysis and discussion is presented in section IV. The paper concludes with its general findings, recommendation and conclusion.

\section{BACKGROUND AND RELATED WORK}

Many studies have investigated factors, challenges and obstacles that have an influence on adopting e-government in many countries, e.g. Jordan, Bahrain, Qatar, Egypt, Pakistan, Taiwan, Germany and Saudi Arabia such as [4][2][13][1][28][27][30][31][32]. These studies have introduced to which extent such factors impact on adopting egovernment from the perspective of users or governments. The studies have found that most countries lack considering factors and addressing challenges and obstacles which have an influence on adopting e-government and providing it to the fullest. Furthermore, the results have provided only general recommendations for countries' governments to focus attention on all or some of these factors or obstacles without proposing specific solutions. Table 1 shows most factors, challenges and obstacles found in adopting e-government in many countries.

E-government acceptance should highly consider a number of factors which are related to users' needs, e.g. culture, education, usability and accessibility, and not just interest in implementing good infrastructure for ministries, e.g. availability of hardware, networks and servers [4][2][13][1]. In Saudi Arabia, [2][4] have studied challenges and obstacles affecting e-government adoption. The main obstacles have 
been found: availability, education level, trust, accessibility and usability [2][4].

\section{TABLE I. CHALLENGES AND OBSTACLES IN E-GOVERNMENT}

\begin{tabular}{|l|l|l|}
\hline Factors & Explanation & References \\
\hline $\begin{array}{l}\text { Infrastructure, e- } \\
\text { readiness, } \\
\text { availability }\end{array}$ & $\begin{array}{l}\text { To what extent the major IT } \\
\text { infrastructure facilities, e.g. hardware, } \\
\text { software and skills, are ready and } \\
\text { available to all users and government } \\
\text { sectors }\end{array}$ & $\begin{array}{l}{[2],[4],[27],} \\
{[30]}\end{array}$ \\
\hline $\begin{array}{l}\text { Privacy, security, } \\
\text { trust }\end{array}$ & $\begin{array}{l}\text { To what extent the government } \\
\text { provides a secure e-transaction and } \\
\text { ensures user information privacy. }\end{array}$ & $\begin{array}{l}{[1],[2],[4],} \\
{[30],[31]}\end{array}$ \\
\hline $\begin{array}{l}\text { Service } \\
\text { complexity, ease } \\
\text { of use, } \\
\text { accessibility }\end{array}$ & $\begin{array}{l}\text { To what extent the e-service } \\
\text { design is usable, accessible and } \\
\text { understandable by different citizens. }\end{array}$ & {$\left[\begin{array}{l}\text { To what extent the information } \\
{[30],[32],[27],}\end{array}\right.$} \\
\hline $\begin{array}{l}\text { Service quality, } \\
\text { website quality, } \\
\text { compatibility }\end{array}$ & $\begin{array}{l}\text { and content of the e-government } \\
\text { websites satisfy different citizens and } \\
\text { are compatible with a unified design } \\
\text { framework. }\end{array}$ & {$[27],[30],[32]$} \\
\hline $\begin{array}{l}\text { Culture, } \\
\text { education, } \\
\text { Internet and } \\
\text { computer skills }\end{array}$ & $\begin{array}{l}\text { Country culture, religion, } \\
\text { education level of different citizens, } \\
\text { and computer skills all have an impact } \\
\text { as social factors in adopting e- } \\
\text { government }\end{array}$ & {$\left[\begin{array}{l}\text { [1], [2], [4], } \\
{[31]}\end{array}\right.$} \\
\hline Awareness & $\begin{array}{l}\text { To what extent a country's } \\
\text { government spreads the awareness }\end{array}$ & {$[30]$} \\
\hline
\end{tabular}

The ability to read and write is one of the important factors which allow individuals to integrate in society and digital society, and exchange knowledge and information. The United Nations Educational, Scientific and Cultural Organization (UNESCO) has defined literacy as "the ability to read, write and understand short written sentences in everyday life". Being unable, or finding it difficult, to read or write is a kind of disability which mediates e-inclusion and accessibility of egovernment websites [15].

UNESCO provides great effort and many programmes to eliminate illiteracy in many countries. UNESCO considers literacy to be very important, especially in rapid development and usage of technology in most of our lives [15]. However, still there are groups of illiterate people in many countries according to statistics. According to UNESCO Day 2014, "there are still 781 million adults and 126 million youths who cannot read or write a simple sentence" [15].

Recently, an article was published in Al Eqtisadiah newspaper in Saudi Arabia which states that the number of illiterate people in Saudi Arabia amounted to 1.2 million of the population by the end of 2013; this number forms $5.6 \%$ of the population [8]. The highest rate of illiteracy was in elderly people between 50 and 75 years of age, with the rate at about $40 \%$ regarding total illiterate people who are aged above 15 years [8]. This group of users need to utilise e-services. However, there are only a few studies that have addressed and developed a suitable interface for illiterate and semi-literate users to improve their ICT projects in developing countries such as [3][5][7][9][11][14]. Addressing a problem related to people who have difficulty in accessing ICT projects is not just a hardware problem, but needs to consider other factors, e.g. how to design and provide information presented in ICT projects [7].

Medhi et al. defined a text-free interface as the "liberal use of graphics and photographs for visual information, and voice for providing information normally provided via text" [11]. Thus, a text-free interface is a replacement for textual content with expressive photographs, drawing images, and audio. It has been shown that an interface without text can be useful and preferable by users unable to read text (illiterate) and with a low level of education (semi-literate) [11][9][16]. Nevertheless, most illiterate people used mobile phones, were familiar with number pads, and were able to identify numbers [11][9]. This implies that a text-free user interface can be usable by illiterate users when containing numerical text.

The process of developing an interface for illiterate and semi-literate users includes observing and understanding users in specific contexts, as well as analysing and gathering information, and includes these perspectives in the design [11]. Medhi et al. and other studies have proven that involving and understanding illiterate users' needs in a design process can help to recognise problems during interface development and selecting the more effective and expressive types of pictures and other types of media [11][6][3][5]. Due to illiterate people often having a different system of thinking, some pictures may be interpreted completely differently, depending on the level of education of the person and culture [7].

Thatcher et al. have developed an ATM icon-based interface in respect of helping illiterate bank customers [16]. Choosing the best icons and pictures to translate the intended meaning of content depends on the community culture, psychology and religion of illiterate users [7][3]. However, Goetze et al. and Thatcher et al. have found that most of the pictures have the same interpretation in different countries, whereas native language is understandable by only a specific country or region [7][16]. This led to concluding that an iconbased interface is better than a speech-based interface for illiterate people, especially in countries with many different languages, dialects and accents [16]. In addition, the human mind responds to images and it is very easy for a user to understand a particular text in the form of pictures [7].

Ávila et al. and Medhi et al. have shown that illiterate people can interpret both realistic pictures and drawing (cartoon) images [7][3]. Choosing between these two types of icons depends on the context of the task and system [3]. Designers can use realistic pictures when focusing on the credibility or creating a direct connection with a particular concept in the real world [3]. However, they can use drawing images when focusing on representing a general concept or they need to take advantage of the flexibility to draw concepts rather than realistic pictures [3]. Insertion of expressive pictures and icons in conjunction with text can be beneficial for functionality and lowly literate people. Friscira et al. have shown that keeping text with pictures will be better for low illiteracy, due to removing text not allowing low literates to discover and encourage them to learn reading and writing [6].

The VideoKheti mobile system project has shown the benefits of using multimodal interaction when designing a text- 
free interface [5]. Multimodal systems can offer a flexible, efficient and usable environment for lowly literate users [5]. Therefore, it provides many ways for lowly literate users to interact through input modalities, such as speech and touch, in addition to receiving information through the system through output modalities, such as speech synthesis, smart graphics and other modalities, opportunely combined [9]. This method of HCI allows lowly literate and novice users to choose their preferred style of interaction [9]. VideoKheti has been built on multimodal interfaces and the first system is targeted at lowly literate users, which combines touch, graphics, and speech input on a mobile interface and is completely text-free [5]. However, it has examined this kind of interface with semiliterate people and applied the project to mobile phones only.

Sherwani et al. have developed a telephony system to help illiterate people by using speech recognition technology and comparing it with touch technology to access information in a healthcare system [14]. However, the adoption of a speech recognition interface for illiterate users' needs considerable effort and to be well designed in order to be better and more effective than a touch interface for both low-literacy and higher-literacy users [14].

Earlier studies have been focused on an innovative way to address illiteracy constraints through designing a user interface suitable for the illiterate and semi-literate population [3][5][6][11][14]. It will adopt and use icons and images only in designing the text-free interface [16]. As mentioned before, the advantage of icons and images is that they are more understandable by different countries and regions with a variety of languages and dialects, whereas the disadvantage is how to refine and select the best icons and images to reflect the intended meaning of instructions or tasks in a specific system. Medhi et al. have suggested using voice feedback as help instructions in order to explain some instructions or tasks [11], designing a user interface using text with icons and images in order to encourage semi-literate users to learn reading or guess written content with the help of icons [6][3]. Nevertheless, the text will not help illiterate people as they never read or write. A speech-based user interface will be designed as an interaction method for lowly literate people [14]. However, it needs to be well designed due to different dialects and accents, particularly in larger countries.

\section{RESEARCH QUESTION AND METHODOLOGY}

This research aims to examine the impact of text-free user interface on usability while illiterate and semi-illiterate use eservices. This can be achieved after proposing a consolidated framework, which will adopt culture, users' needs and usability guidelines, to develop text-free user interface for a local eservice. A prototype will be developed taking into account the proposed consolidated framework. The proposed prototype will be evaluated in terms of its usability with illiterate and semiilliterate users through proper usability evaluation methods.

\section{A. selection and Development of text-free web-based system:}

An e-government web-based system has been chosen for this research where it has to be used by the all citizen her in Saudi Arabia so illiterate and semi illiterate people have to use such a system. In order to develop the prototype; three steps have to be considered: images and icons development, images' should meet usability guidelines and cultural and users' needs should be also considered. Firstly, all images and icons have been designed for the purpose of the project and to fit with the selected e-service functions except the common icons and images such as cancellation icons and others. Secondly, images usability guidelines have been taken into considerations such as meaningfulness, styling quality, localability, message quality and metaphor. Thirdly, culture and users' needs have been also considered such as the images should be acceptable with Saudi culture. It will also reflect users' needs while using the proposed system. The prototype of the selected system has been developed taking into consideration the aforementioned steps consulting 2 experts to ensure a professional development has been taken into account.

Usability evaluation is expected to be carried out after ensuring the prototype has been developed and its ready for evaluation to answer the research question. Two usability evaluation methods have been used in this research; usability testing and heuristic evaluation. the UT method needs users participation where heuristic evaluation needs experts to be conducted. Therefore, both methods have been chosen for this research in order to eliminate any risk of ignoring any type of “ user" or " expert" point of view.

Usability testing aimed at gathering qualitative and quantitative data by observing the users' performance and user satisfaction after finishing the test session, as well as finding usability problems potentially encountered by illiterate/semiliterate users. This method needs representative users to perform predefined tasks. Participants recruitment, task selection, test environment and measures will be discussed.

\section{B. Participants recruitment:}

Saudi illiterate/semi-literate users were the targeted audience of both genders. Neilson found that five users are enough for user testing through formative usability evaluation; however, 15 users help to identify more problems at the first evaluation [20]. Dumas and Redish have pointed out that 6-12 users are sufficient to conduct user testing [21]. Thus, in this testing, 15 participants have been recruited. Moreover, user consent was taken from users. The pre-test questionnaire was filled out by the users.

\section{Task selection:}

The tasks have been selected based on the four selected services which have been designed for the purpose of the project. Each service is represented as a user task, as recommended by [34] [21].

\section{Test environment:}

An appropriate location has been prepared and organised to conduct an effective test session. The selected location should be quiet and comfortable for every participant. The observer sat away from the participant as not to interrupt his/her attention while performing the given tasks as its recommended by [34][21].

\section{E. Usability measurements:}

The usability of a software, website or product is measured by to what extent it is easy to use for the targeted users [19]. 
Five metrics have been identified by Jakob Nielsen to evaluate the ease of use or usability: learnability, efficiency, memorability, errors and satisfaction [19]. Memorability is measured by how users become proficient when facing the design after a period of time. Thus, it has been excludes due to the long time needed for the evaluation. Learnability is calculated by task completion when the user performs the task the first time [19]. Task completion has been determined by monitoring the user during the task using three rates proposed by Jakob Nielsen $(\mathrm{S}=$ Success, $\mathrm{F}=$ Failure, $\mathrm{P}=$ Partial success) [22]. Success indicates the user terminating the task without errors, Partial success means the user succeeding in performing the task with error(s), and Failure means the user giving up finishing the task [22]. The efficiency is checked by the time the user takes to complete the task after learning the design [19]. For that, the test has been recorded using Screen Recorder software, which helps to measure the efficiency (time on completion of task). The errors measure is calculated by the number and frequency of errors encountered by the users during the test [19]. Also, Screen Recorder software was used to identify user errors through performing each task. Satisfaction is evaluated by to what extent the user enjoyed and was interested in using the design [19]. User satisfaction has been measured by asking the user orally after finishing the tasks using a 5-point Likert scale (very satisfied, satisfied, neutral, dissatisfied, and very dissatisfied).

Heuristics evaluation was organised and prepared to ensure effective inspection and results. Preparing the procedure included selection of evaluators, guidelines with detailed checklists, stating evaluation materials and instructions, and piloting the guidelines and checklists to ensure its compatibility with the text-free web-based system before starting the real evaluation.

\section{F. Selection of heuristics evaluation guidelines:}

The most frequent set of heuristics used in evaluating a user interface has been proposed by Nielsen [24]. It consists of 10 heuristics which are used in text-free user interface evaluation [3]. Nevertheless, a new text-free interface needs additional principles or guidelines in conjunction with Nielsen's heuristics. For this reason, Nielsen's 10 heuristics have been extended with text-free principles proposed by Medhi et al. during her field study [11]. These principles have developed as guidelines for a text-free user interface. Furthermore, Flexibility and Minimalist Design principles in Nielsen heuristics have been excluded because it intended to evaluate the ability of expert users to accelerate his interaction with the interface by using shortcuts or an alternative way of interaction. This heuristic is not suitable for illiterate/semiliterate users because they need a few steps and settings in order to reduce the potential user errors. In addition, there is little knowledge and experience of illiterate/semi-literate users in using a computer; thus, there is no room for adjusting interface settings. The following list has been used which is a combination of Nielsen heuristics [24] and Medhi text-free principles [11]:

1) Visibility of System Status

2) Match Between System and the Real World

3) User Control and Freedom
4) Consistency and Standards

5) Help Users to Recognise, Diagnose, and Recover from Errors

6) Error Prevention

7) Recognition Rather Than Recall

8) Aesthetic and Minimalist Design

9) Help and Documentation

10)Avoid text (but using numbers may be okay).

11)Use semi-abstracted graphics, photorealism with deeper interaction

12)Pay attention to subtle graphical cues. User response may depend on psychological, cultural or religious biases.

13)Provide voice feedback for all functional units

\section{G. Selection of evaluators:}

Five evaluators were recruited (two female and three male) after taking their consent as recommended by [17]. The evaluators have studied a usability analysis and testing course and have little experience in using a text-based website version.

\section{H. Evaluation materials and steps:}

Afterwards, a heuristics evaluation sheet was prepared with 13 selected heuristics. A number of checklists have been listed in order to help the evaluator to assess the interface against every heuristic. The evaluation sheet was submitted by e-mail to everyone in order to ensure conducting the evaluation independently to guarantee unbiased results [25]. The evaluation sheet included the goal and objectives of assessment and the website tasks without providing any guide or aid as to how to use the design. Furthermore, it was requested from each evaluator to spend some minutes exploring the interface and then review it to fill out the sheet, find problems and provide their comments. The problems found during heuristics evaluation have integrated with user testing problems in a single table (in the next section).

\section{Piloting the experiment:}

A pilot experiment of heuristics checklists has been done by an independent evaluator. The importance of piloting the heuristics checklists is to guarantee that it is adequately clear and appropriate to the text-free interface [25]. In the pilot experiment, the evaluator has achieved all heuristics evaluation steps and procedures. The results of this evaluation are excluded from findings due to there being discussion and clarification with the evaluator.

\section{J. Severity rating:}

Problem severity helps to rank the seriousness of the problem to the targeted users during using the website. Using Jakob Nielsen's severity rating, all problems gathered and observed by two usability methods have been returned to the five heuristics evaluators to rank the severity of each problem [26]. The severity rating scale from 0 to 4 is as follows: $0=\mathrm{I}$ don't agree that this is a usability problem at all; $1=$ Cosmetic problem only: need not be fixed unless extra time is available on the project; $2=$ Minor usability problem: fixing this should be given low priority; 3 = Major usability problem: important to fix, so should be given high priority; $4=$ Usability catastrophe: imperative to fix this before the product can be 
released [26]. Each evaluator did the rating alone and then computed the average of rates for each problem [26].

\section{DATA ANALYSIS AND DISCUSSIONS}

This section includes analysis of data collected from two usability evaluation methods which have been selected in this project. Starting with finding all problems from these methods, a comparison between types of problems has been discovered by each method. Thereafter, analysis data was gathered from usability testing in order to assess and find the overall user usability of the text-free web-based system.

\section{A. User Testing and Heuristics Evaluation Problems:}

After running user testing and heuristics evaluation, this section discusses what problems have been detected, the number and frequency of problems found by each method, common problems discovered by both, and the types of problems in terms of the impact of the problem on user usability. The following table (Table 3 ) shows the number of problems discovered by heuristics evaluation and usability testing and the number of common problems:

TABLE II. NUMBER OF PROBLEMS

\begin{tabular}{|l|c|c|}
\hline Evaluation Method & $\begin{array}{c}\text { No. of } \\
\text { Problems }\end{array}$ & $\begin{array}{c}\text { No. of Common } \\
\text { Problems }\end{array}$ \\
\hline Heuristics Evaluation & 17 & \multirow{2}{*}{4} \\
\hline Usability Testing & 9 & \\
\hline Total no. of problems & 22 & \\
\hline
\end{tabular}

Overall, 22 distinct problems have been discovered from both usability testing and heuristics evaluation. Usability testing has revealed five distinct problems, heuristics evaluation has found 13 distinct problems, and four problems were common.

\section{B. Discovered problems based on frequency:}

From the extracted problems, the number of observations have been discovered. The evaluation found that the highest frequency was in problems which related to skills needed in using a computer (using a dropdown menu and check boxes). Most of the illiterate/semi-literate users do not use a computer. The usability tester observed that illiterate/semi-literate users tend to click on-based tasks.

Regarding the images and icons design problems were in second place with less frequency. This was according to the images and icons feature which was assumed before in the consolidated framework. Notice that only images and icons belong (some concepts or website functions need help in conjunction with images such as a voice or video, as there is no agreement to interpret the same exactly intended meaning by all people), e.g. the image of the Reporting Missing Documents service has a problem. Although there is help in the form of a voice with the image, it may need to add the voice when the mouse is over an image instead of a separate icon for a voice under the image. That is easier for illiterate/semi-literate users, as observed in the map during the evaluation.

The other images and icons which belong to other features have been recognised by users. However, regarding the feature that is assumed (illiterate/semi-literate users can recognise numbers), only two users out of 15 can't read numbers.

\section{Discovered problems based on severity:}

Heuristics evaluation found a usability catastrophe and mostly major and cosmetic problems. It revealed the problems related to different aspects in overall web design, e.g. validation problems, error prevention problems, page title, and space between icons which are not detected or overlooked by real users. The major problems by heuristics may have an effect on users' usability after a period of time of interaction with the design. Usability testing discovered major and minor problems frequented by users. This leads to heuristics evaluation considered a complement to usability testing and these two usability methods can provide exhaustive usability evaluation [33]. Table 3 shows the discovered problems based on severity.

TABLE III. TYPE OF PROBLEMS BASED ON SEVERITY

\begin{tabular}{|l|c|c|c|l|}
\hline $\begin{array}{l}\text { Type of } \\
\text { Problem }\end{array}$ & $\begin{array}{l}\text { \# of HE } \\
\text { Problems }\end{array}$ & $\begin{array}{l}\text { \# of UT } \\
\text { Problems }\end{array}$ & $\begin{array}{l}\text { \# of common } \\
\text { Problems }\end{array}$ & Total \\
\hline catastrophe & 1 & - & - & 1 \\
\hline Major & 5 & 1 & 2 & 8 \\
\hline Minor & 1 & 2 & 2 & 5 \\
\hline Cosmetic & 6 & 2 & - & 8 \\
\hline
\end{tabular}

\section{Usability Testing:}

The data collected from usability testing have been used to assess the text-free web-based usability according to usability metrics: learnability, efficacy and user errors. As mentioned before, the testing was carried out on 15 (seven male and eight female) participants. They have the following profile characteristics as shown in Table 4:

TABLE IV. Participants' Profile Charictersitics

\begin{tabular}{|c|l|l|l|l|}
\hline Characteristics & Response & $\begin{array}{c}\text { Male } \\
\text { (Percentage) }\end{array}$ & $\begin{array}{c}\text { Female } \\
\text { (Percentage) }\end{array}$ & Total \\
\hline \multirow{5}{*}{ Age } & $20-30$ & $0(0 \%)$ & $1(12.5 \%)$ & $1(6.6 \%)$ \\
\cline { 2 - 5 } & $31-40$ & $2(28.6 \%)$ & $2(25 \%)$ & $4(26.6 \%)$ \\
\cline { 2 - 5 } & $41-50$ & $4(57.1 \%)$ & $3(37.5 \%)$ & $7(46.6 \%)$ \\
\cline { 2 - 5 } & $\begin{array}{l}51 \text { or } \\
\text { more }\end{array}$ & $1(14.3 \%)$ & $2(25 \%)$ & $3(20 \%)$ \\
\hline \multirow{3}{*}{$\begin{array}{l}\text { Educational } \\
\text { Level }\end{array}$} & Illiterate & $4(57.1 \%)$ & $3(37.5 \%)$ & $7(46.6 \%)$ \\
\cline { 2 - 5 } & $\begin{array}{l}\text { Semi- } \\
\text { literate }\end{array}$ & $3(42.9 \%)$ & $5(62.5 \%)$ & $8(53.3 \%)$ \\
\hline \multirow{5}{*}{ Computer Skills } & Excellent & $0(0 \%)$ & $0(0 \%)$ & $0(0 \%)$ \\
\cline { 2 - 5 } & Good & $2(28.6 \%)$ & $1(12.5 \%)$ & $3(20 \%)$ \\
\cline { 2 - 5 } & Bad & $3(42.9 \%)$ & $4(50 \%)$ & $7(46.6 \%)$ \\
\cline { 2 - 5 } & No skills & $2(28.6 \%)$ & $3(37.5 \%)$ & $5(33.3 \%)$ \\
\hline
\end{tabular}

\section{E. Learnability (Task Completion):}

As observed in the success level chart, two users failed in completing Task \#1, as they were unable to identify the way of selecting the services (small check box). These users were aged 51 and above; moreover, they did not have experience with a computer and bad experience skills in using a smart phone.

In Task \#2, four users failed in performing the task on account of the Reporting Missing Documents icon on the home page not translating the intended meaning. They have clicked on booking an appointment at Civil Affairs, and their commentary does this service in the government office. They also did not try to click on voice icons. Furthermore, they performed Task \#2 as a first or second task according to the assigned task order. 
Furthermore, four users failed in Task \#4 because they failed in how to enter the date using the dropdown menu. Note that all of these users did not have or have bad computer skills.

The success rate was calculated as Jakob Nielsen suggested. Partial success (p) took $50 \%$ of success, was added to the success ( $\mathrm{S}$ ) case, and then divided the value over the overall attempts as follows $\left(\mathrm{S}+\left(\mathrm{p}^{*} 0.5\right) / \mathrm{No}\right.$ of attempts) [22]. The overall success rate of the design from 60 attempts is $75 \%$. Jakob Nielsen found that most website success rate scores are less than $50 \%$ if the user is dealing with design the first time [22]. Additionally, Jeff Sauro pointed out $78 \%$ as a measurement for the success rate. This percentage was an average success rate found in 1200 different areas and the design of software and website tasks with expert and novice users [29]. Ismail has assessed the usability of educational computer games for children which have the same characteristics of our design in respect of using images and children not proficient in reading. Their system achieved a $73.18 \%$ success rate [28]. The result of $75 \%$ is considered reasonable and good due to a score above $50 \%$ compared with Nielsen, as the users interact with the system the first time, which is close to Ismail, finding $73.18 \%$ when compared to a similar design and characteristics. It is also close to Jeff Sauro's success rate measurement, although his results are from different types of users and software domains.

\section{F. Efficiency (Time on Task):}

During the usability testing session, the observed efficiency of the success completion task increases based on the task order. The time of every task decreases if the task has been performed by a user as the third or fourth task in order regardless of the user profile or characteristics. This is due to the user becoming more familiar with the design and the design becoming learnable after performing more than two tasks [19]. In these results, Task \#1 has been excluded because only one success case happened.

The geometric means have been considered in computing the average time for each task, as recommend by Jeff Sauro and Jakob Nielsen [23]. The geometric mean is better in order. A very big value can skew the result in an arithmetic mean, especially if the sample size is less than 20 [23]. As mentioned before, excluding Task \#1, Task \#4 has the highest geometric mean due to having the highest number of steps to perform it relative to other tasks. Table 5 displays the geometric mean for Task \#2, Task \#3 and Task \#4:

TABLE V. GeOMETRIC MeAn of TASK Time

\begin{tabular}{|l|l|}
\hline Task & Geometric Mean $($ sec.) \\
\hline Task \#2 & 69 sec. \\
\hline Task \#3 & 84 sec. \\
\hline Task \#4 & 117 sec. \\
\hline
\end{tabular}

\section{G. Number of errors:}

Most problems have been frequented in Task \#1 (roughly $45 \%$ (19 user errors from 42) of the total frequency rate during usability testing). This problem related to computer skill (selecting from check boxes). Meanwhile, Task \#3 has recorded the lowest frequency rate of $2 \%$ (one user error from 42) during usability testing due to no computer skills needed (depending on click or touch). Moreover, Task \#2 and Task \# contain a dropdown menu that needs some skills in using a computer. The only user error with the most frequency related to image and icon design was the Reporting Missing Documents icon. The non-understandable meaning of this icon while using a dropdown menu error in Task \#2 has influenced an increase in the number of errors in Task \#2 after Task \#1 is in place.

\section{H. Satisfaction:}

The satisfaction was measured by one question to assess the overall user experience and pleased after using the text-free interface. Single Ease Questions (SEQs) have been used to measure the overall user experience and satisfaction with the design (as recommended by Jeff Sauro) being easy to answer and collect, particularly for illiterate/semi-literate users [34]. We used a 5-point Likert scale in the form, and explained each point scale to the user to select one of them. The answer was taken orally by the observer after the test session. Although some of the users succeeded and some had errors, all of the users had a positive response and a willingness to use the textfree interface if available on government websites.

\section{MOST IMPORTANT FINDINGS AND PROJECT CONTRIBUTION}

Conducting such research offers different findings and contributions. These can be divided into different categories, such as research findings, recommendations, and contributions.

\section{A. Research Findings:}

As discussed in the beginning of this project, adoption of egovernment requires decision makers to pay more attention to users' needs. It can be noticed that previous literature in many countries, particularly in Saudi Arabia, has not considered some of the factors which would help them to achieve success in implementing e-government. These factors are related to users, such as availability, accessibility, usability and different education levels.

Furthermore, it can be also noticed that a few researchers have conducted and proposed solutions for illiterate/semiliterate users in different developing countries, such as India, Brazil and Switzerland [7][6][3]. To the best knowledge of the author's knowledge, there are no proposed solutions related to user interfaces which have been introduced in Saudi Arabia for people with a low level of education; in addition, the user interfaces proposed in other countries have different representations, such as using icons only [16], icons with text for semi-literate users [6][3], speech [14] or multimodal representations on a mobile phone for semi-literate users only [5].

Thus, this project can be considered proposing a solution to help governments and other organisations in solving availability, accessibility and usability of web-based system content for low education level problems. This solution adopts a text-free user interface by using a combination of icons, images and audio, proposing a consolidated framework in order to accelerate the development process of appropriate and understandable icons and images.

This project has found that text-free interfaces have influences on user performance and satisfaction when illiterate 
and semi-literate people use the system. The users' performance has increased users' learnability and efficiency. The design success rate has achieved $75 \%$, which can be considered positive as illiterate/semi-literate users have encountered the interface the first time when compared with the achieved results of [22] and [29]. This group of users cannot be considered expert users. Additionally, users' learnability has been affected positively where users did not need more time after they performed the first task. This concludes that the text-free interface is easy to learn and illiterate/semi-literate users became familiar with the interface after performing one or two tasks. It has been observed that illiterate/semi-literate users are willing to accept and use egovernment web-based systems if they consider their needs and become text-free interfaces. This result is in line with [16] and [11].

\section{B. Recommendations:}

It can be recommended that adoption of text-free user interfaces can positively influence the usability of egovernment systems while illiterate/semi-literate users use them. Moreover, it can be also recommended that user interface design should be as simple as possible in respect of interaction methods, avoiding complex task actions, small images, icons and interface controls, and adopting click- or touch-based tasks as suggested by references [5] and [6] and mouse-over actions as pointed out by Medhi [11].

Furthermore, it is highly recommended that designers and developers use the consolidated framework as it helps in the design of images and icons and in developing effective and efficient images and icons for different websites, cultures and countries. Image usability guidelines, countries' culture, and proposed image features during usability evaluation methods have shown their adequacy to accelerate reaching or closing understandable images and icons without iterative design with real users which happened with Thatcher and Medhi [16] [11]. Moreover, image features were based on using truthfulness and clear and visible images and icons, e.g. real objects in life, globally known things and countries' environment and culture. The images do not matter if represented in a photographic or abstraction way, but they should be credible and intelligible and have a direct meaning to the intended concept as found by Ávila [3]. Additionally, using voices can be helpful in conjunction with concepts which do not have agreement representation or images. In [16], it has been mentioned that during his experiments to develop comprehensible icons for all users, it was not necessary for all icons to be $100 \%$ understandable by all users, as users could understand them after continuing using the interface [16].

\section{Conclusion and Future Work:}

Current and relevant literature has been reviewed in respect of developing a text-free interface for illiterate and semiliterate people in many countries. Moreover, the literature review covered discount usability evaluation methods and compared these methods. The project has developed a webbased system for government services in Saudi Arabia. The project suggests a consolidated framework which is expected to include users' needs, cultural factors, and usability guidelines which can be used for developing any text-free interface. Therefore, all of the selected e-services will be developed to be text-free interfaces, applying usability testing and heuristic evaluation on a developed design in order to answer the research question. The project found that the textfree interface has an influence on improving illiterate/semiliterate usability of e-government systems. The text-free interface should be designed in such a way as to be simple and understandable by these kinds of users according to their country, culture and website domain.

For future work, this project can be extended to redesign the interface to solve the problems which were discovered as major problems during usability evaluation. Furthermore, transfer another service on the Ministry of Interior websites. The following table contains major problems discovered during usability evaluation and recommendations for a future design. This research can be applied to different domains, e.g. healthcare, or different countries and cultures when using the same proposed image features or adding new features based on the user's needs who they targeted. It can be also compared with the traditional e-services. It can also be extended to target different groups of users such as disabled people.

\section{ACKNOWLEDGMENT}

Thanks to Dr. Qazi Mudassar Ilyas and Dr. Amna Asif Khan for their valuable feedback while conducting this research.

\section{REFERENCES}

[1] Alomari, M., Sandhu, K., \& Woods, P. (2010). Measuring social factors in e-government adoption in the Hashemite Kingdom of Jordan. International Journal of Digital Society (IJDS), 1(2), 163-172.

[2] Alshehri, M., Drew, S., \& Alfarraj, O. (2012). A comprehensive analysis of e-government services adoption in Saudi Arabia: Obstacles and challenges. Higher education, 6, 8-2. LITERACY DATA SHOW PERSISTENT GENDER GAP.

[3] Ávila, I., \& Gudwin, R. (2009). Icons as helpers in the interaction of illiterate users with computers. Proc. of the Interfaces and Human Computer Interaction, 20-22.

[4] Basamh, S. S., Qudaih, H. A., \& Suhaimi, M. A. (2014). E-Government Implementation in the Kingdom of Saudi Arabia: An Exploratory Study on Current Practices, Obstacles \& Challenges. International Journal of Humanities and Social Science, 4(2), 296-300.

[5] Cuendet, S., Medhi, I., Bali, K., \& Cutrell, E. (April 2013). VideoKheti: Making video content accessible to low-literate and novice users. In Proceedings of the SIGCHI Conference on Human Factors in Computing Systems (pp. 2833-2842). ACM.

[6] Friscira, E., Knoche, H., \& Huang, J. (March 2012). Getting in touch with text: Designing a mobile phone application for illiterate users to harness SMS. In Proceedings of the 2nd ACM Symposium on Computing for Development (p. 5). ACM.

[7] Goetze, M., \& Strothotte, T. (2001). An approach to help functionally illiterate people with graphical reading aids. In Smart Graphics Symposium UK (pp. 21-23).

[8] http://www.aleqt.com/2015/02/10/article_929782.html, viewed on $15 / 02 / 2015$

[9] Kumar, R. (2011/2012). Graphical User Interface: A GUI based application for illiterate/semi-literate Indian construction laborers, in University of Leeds.

[10] Kumar, S., Karyamsetti, R., Ratala, R., Kaur, A., \& Tejosh, S., Human Computer Interaction for Illiterate and Semi-illiterate Users to Access Egovernment Application.

[11] Medhi, I., Sagar, A., \& Toyama, K. (2007c). Text-Free User Interfaces for Illiterate and Semiliterate Users, Information Technology for International Development, 4(1), 37-50. 
[12] Nordberg, C. (2010). Exploring the text free interface for illiterate users: Designing an icon-based prototype for mobile phones.

[13] Ovais Ahmad, M., Markkula, J., \& Oivo, M. (2013). Factors affecting egovernment adoption in Pakistan: a citizen's perspective. Transforming Government: People, Process and Policy, 7(2), 225-239.

[14] Sherwani, J., Palijo, S., Mirza, S., Ahmed, T., Ali, N., \& Rosenfeld, R. (April 2009). Speech vs. touch-tone: Telephony interfaces for information access by low literate users. In Information and Communication Technologies and Development (ICTD), 2009 International Conference (pp. 447-457). IEEE.

[15] UNESCO Institute for Statistics, http://www.uis.unesco.org/literacy/Pages/literacy-day-2014.aspx, 2014.

[16] Thatcher, A., Mahlangu, S., \& Zimmerman, C. (2006). Accessibility of ATMs for the functionally illiterate through icon-based interfaces. Behaviour \& Information Technology, 25(1), 65-81.

[17] Hertzum, M., \& Nielsen, J. (2001). The Evaluator Effect: A Chilling Fact About Usability Evaluation Methods. International Journal of Human-Computer Interaction, 15(1), pp. 183-204.

[18] Rubin, J., \& Chisnell, D. (2006). Handbook of Usability Testing, Second Edition: How to Plan, Design and Conduct Effective Tests: Wiley Publishing, Inc.

[19] Nielsen, J. (2003). Usability 101: Introduction to usability.

[20] Nielsen, J. (2000). Why you only need to test with 5 users.

[21] Dumas, J. S., \& Redish, J. (1999). A practical guide to usability testing. Intellect Books.

[22] Nielsen, J. (2001). Success rate: The simplest usability metric. Jakob Nielsen's Alertbox, 18.

[23] Measuringu, "Average Task Times In Usability Tests: What To Report?", [Online], http://www.measuringu.com/average-times.php, Jeff Sauro, April 21, 2010.

[24] Nielsen, J. 10 Usability Heuristics for User Interface Design, 1995, accessed: November 12, 2014. [Online]. Available: http://www.nngroup.com/articles/ten-usability-heuristics/
[25] Nielsen, J., \& Molich, R. (March 1990). Heuristic evaluation of user interfaces. In Proceedings of the SIGCHI Conference on Human Factors in Computing Systems (pp. 249-256). ACM.

[26] Nielsen, J. (1995). Severity ratings for usability problems. Papers and Essays, 54.

[27] Mariam Rehman, Vatcharaporn Esichaikul, \& Muhammad Kamal (2012). "Factors influencing e - government adoption in Pakistan", Transforming Government: People, Process and Policy, Vol. 6, Iss: 3, pp. 258-282.

[28] Ismail, M., Diah, N. M., Ahmad, S., Kamal, N. A. M., \& Dahari, M. K. M. (June 2011). Measuring usability of educational computer games based on the user success rate. In Humanities, Science \& Engineering Research (SHUSER), 2011 International Symposium (pp. 56-60). IEEE.

[29] Measuringu, "10 Essential Usability Metrics", [Online], http://www.measuringu.com/average-times.php, Jeff Sauro, November 30, 2011.

[30] Abdel-Fattah, M. A. K. (June 2014). Factors Influencing Adoption and Diffusion of e-Government Services. In European Conference on eGovernment (p. 1). Academic Conferences International Limited.

[31] Meftah, M., Gharleghi, B., \& Samadi, B. (2015). Adoption of EGovernment among Bahraini Citizens. Asian Social Science, 11(4), p. 141.

[32] Liang, S. W., \& Lu, H. P. (2013). Adoption of e-government services: An empirical study of the online tax filing system in Taiwan. Online Information Review, 37(3), 424-442.

[33] Paz, F., Paz, F. A., Villanueva, D., \& Pow-Sang, J. A. (April 2015). Heuristic Evaluation as a Complement to Usability Testing: A Case Study in Web Domain. In Information Technology-New Generations (ITNG), 2015 12th International Conference (pp. 546-551). IEEE.

[34] Measuringu, "If You Could Only Ask One Question, Use This One", [Online], http://www.measuringu.com/blog/single-question.php, Jeff Sauro, March 2, 2010. 